\title{
Challenges to Achieving Accurate Three-Dimensional Process Simulation
}

\author{
M. E. Law \\ Florida Integrated Research in Silicon Technology, Department of Electrical \\ Engineering, University of Florida \\ 339 Larsen Hall, Gainsville, FL 32611, USA
}

\begin{abstract}
This paper will identify the reasons three-dimensional process simulators are not widely available, when three-dimensional device simulators are widely available. There appear to be four major obstacles; metrology, models, numerics, and structural barriers. Each of these will be discussed and possible solutions will be provided.
\end{abstract}

\section{Introduction}

The TCAD community generally recognizes that sub micron MOS and bipolar devices require three-dimensional simulation. In addition, DRAM structures and latchup phenomena are three-dimensional problems. For these reasons, a great deal of work has been performed on three-dimensional device simulation. In fact, three-dimensional device simulators are available from both university and commercial sources. Doping and material information for these device simulators is usually developed from either analytic models or two-dimensional process simulation. Three-dimensional process simulation would be useful not only for analysis of three-dimensional structures, but also in providing accurate doping and material information for use with threedimensional device modeling. The accuracy of a device simulation can be no better than the structural input - "garbage in, garbage out." Yet despite the apparent need for three-dimensional process simulation, there are no widely available process simulators, and little activity is focused on their development. Why?

There have been two traditional branches to process modeling. The first major area is in the simulation of surface evolution and lithography. This activity is topologically two-dimensional, but geometrically three-dimensional. In other words, simulators exist to evolve a two-dimensional surface plane in a three-dimensional space. The other activity is bulk simulation, which focuses on material growth and dopant diffusion. This activity requires the solution of a complex, nonlinear system of equations in the materials of interest. For three-dimensional simulation, it requires three-dimensional grid and structure.

For surface simulation, the state-of-the-art is very good. Excellent results have been attained in modeling the surface evolution. For example, the work of the Berkeley group on surface evolution during resist development requires advanced computational 
geometry to evolve the surface without kinks, folds, and loops [1, 2]. An engineering workstation is sufficient for these types of simulations. In addition to simulation, verification of the simulator is also required. For surfaces, this can also be quite advanced. Slinkmann et al., have recently used a scanning force probe microscope to profile the surface of DRAM trench technology [3]. This technique and others allow new models to be developed and characterized effectively. Consequently, these simulators offer three-dimensional capability currently, and will not be discussed further.

The real problem lies with bulk simulation. Bulk three-dimensional process simulation is not currently available. There are three main reasons for this lack of capability. The first major area is measurement techniques. It is not difficult to measure the $\mathrm{I}-\mathrm{V}$ characteristics of a transistor, but it is costly and less reliable to measure a doping profile. The second reason is the numerical cost. Three-dimensional process simulation is thought to be an expensive computation [4]. The final reason is the difficulty in performing process modeling work in universities and company labs.

\section{Challenges}

\subsection{Metrology}

One of the most difficult challenges facing one, two, or three-dimensional bulk process simulation is in obtaining verification of models. Measurement techniques used to investigate doping profiles are not very accurate and for the most part are inherently one dimensional. This limits our ability to verify the predictions of the models in more than one dimension, and limits the scalability of simpler empirical models. This is further complicated by the fact that all of the available analysis techniques are expensive and difficult to perform.

There are three main techniques available to measure dopant profiles. The first is Secondary Ion Mass Spectroscopy (SIMS) which is a destructive technique that makes an etch pit using ion sputtering and examines the backscattered ions. This technique can accurately measure the chemical concentration of the dopant, but can not determine thin dopant layer concentrations very well due to the broadening of the peak. SIMS detection limits place the lower bound on concentration at $10^{16} \mathrm{~cm}^{-3}$, and measurement of doping levels below this is usually not accurate. Because the raw SIMS data is a particle count, the concentration values must be scaled by a dose calibration. This can also result in some uncertainty in the profiles. This technique only gives resolution in the direction of the etch, and therefore is one dimensional only.

Spreading Resistance Profiling (SRP) measures the resistance as a function of depth. SRP measurement systems extract the mobile carrier concentration from the resistance by using mobility model as a function of concentration. The mobile carrier concentration can then be related to the doping concentration. There are several major problems with this technique that limit its accuracy. The first problem is the mobility model, particularly at high concentrations when damage and precipitates can affect the scattering, but are not included in the mobility model. The second effect is carrier spilling and depletion layers that alter the carrier concentration from the doping concentration. These two effects limit SRP to characterization of moderately doped profiles that are not ultra shallow. Related to SRP is capacitance-voltage profiling since it also measures carrier concentrations. This technique can be accurately coupled to Poisson solvers to obtain doping profiles, but is limited to lightly doped layers so that they can be depleted from a surface voltage.

The final measurement technique is junction staining. This technique allows chemical delineation of the junction based on the doping type, and is the only technique that 
can bêt used to examine doping in two dimensions. However, it gives only a single data point - the junction depth. This limits its ability for verification. Some clever structures have been used with junction staining to obtain two-dimensional information [5].

There has been research work on multidimensional measurement techniques. Work has been performed using multiple stains, which allow multiple concentrations to be resolved [6]. This approach makes use of the two-dimensional nature of staining to add additional data beyond just a junction position. Subrahmanyan used this technique to verify two-dimensional simulations of doping profiles near mask edges [7]. GoodwinJohannson has investigated using multiple angled SIMS beams to reconstruct twodimensional doping profiles. This work shows promise, but is limited by the resolution of the SIMS spot. A large spot produces averaging over a wide lateral area [8]. Finally, electrical information can be used to deconvolve two-dimensional profiles, e.g., Khalil and Faricelli [9], but is limited often times by uncertainties in other structural data. This limitation in measurement technology severely hampers development of accurate multi-dimensional simulators. Since it is impossible to verify two-dimensional doping profiles, why bother to compute them in three-dimensions?

\subsection{Models}

In device simulation, there are many levels of simulation complexity available. There are drift-diffusion simulators, energy balance codes, full hydrodynamic solvers, and finally Monte Carlo techniques that use a variety of approximations to the band and scattering problems. This richness of complexity is not available in process simulation. There have been essentially two approaches to bulk simulation; a phenomenological approach, best exemplified by the decision tree simulator PREDICT [10], and a point-defect-based approach, as demonstrated by SUPREM-IV [11, 12]. The phenomenological approach would be the natural tack to take for three-dimensional bulk simulation since it requires less CPU time, but development of these models is delayed by the lack of multidimensional measurement techniques of doping profiles.

This leaves full point-defect-based simulators, which offer the promise of accurate multidimensional simulation due to their complete encapsulation of the full physics of dopant diffusion. These simulations rely on accurate calculation of point defect concentrations that are then used to compute the dopant diffusion. Although conceptually it promises great accuracy, there are two main difficulties. The first is parameterization and second is computation time.

Figure 1 shows the interstitial diffusivity in silicon as a function of temperature and experimentalist. This large scatter in the data does not inspire confidence in the predictive ability of the simulator. Although this experimental scatter can be explained [5], it does point out one of the problems with the point-defect-based simulators. Both point defect types, interstitials and vacancies, can not be measured directly and can only be measured indirectly through their effect on processes, e.g., diffusion. This leads to circular reasoning - there must be excess interstitials because phosphorus diffuses faster, and the reason for the faster diffusion of phosphorus is that there are excess interstitials present. This circular reasoning loop is difficult to break, and has resulted in a great deal of controversy about the point defect behavior in silicon.

Finally, there are a large number of equations to be solved in point-defect-based simulators. Because the approach has always been to include as much physics as possible in the simulation to obtain accuracy, it is often necessary to solve 3-5 partial differential equations to obtain the solution for a single dopant profile. This adds to the burden placed on the numerics of the simulation. 


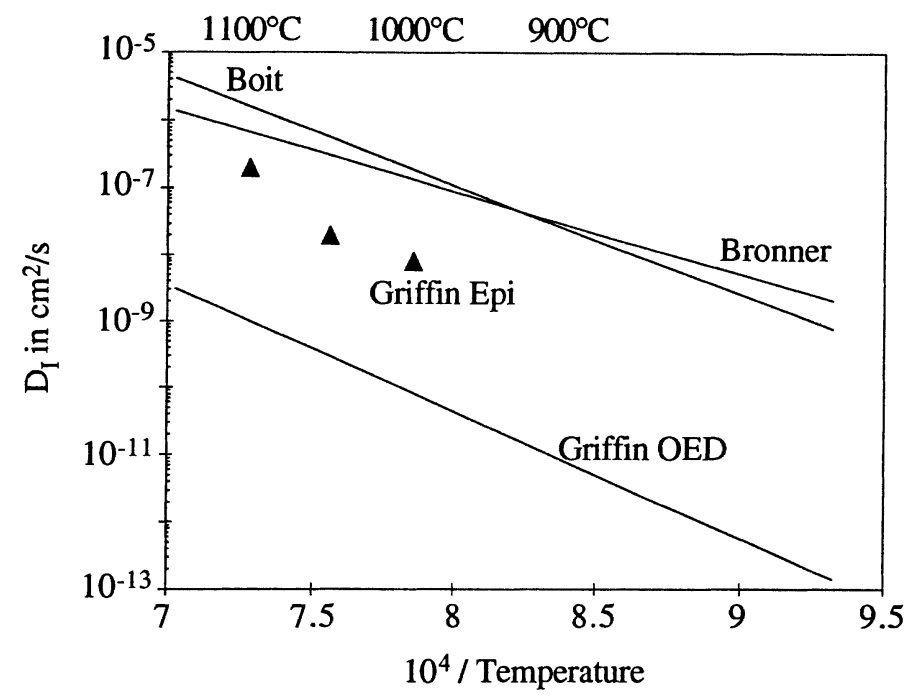

Figure 1: Experimental investigations of the interstitial diffusivity as a function of temperature [5, 13-15]. Different results are obtained under different experimental conditions.

\subsection{Numerics}

There are two major challenges numerically to be solved before three-dimensional process simulation can become a reality. The first involves the linear algebra and the second involves grid generation and adaptation. Both issues must be addressed, but the second is more the province of process simulation than device simulation.

Linear algebra is complicated due to the immense problem size involved. A minimum of roughly one hundred grid points is required to represent a dopant profile to 1 there will be approximately one million nodes. As stated earlier, point-defect-based simulators require three solution variables to solve for a single dopant. The resulting linear sparse system will require approximately three million unknowns. Since solution of linear systems requires approximate solution time on the order of the number of unknowns to the $3 / 2$ power, the computation time required for three-dimensional process simulation is very large, even for super computers. This situation is similar to that for device modeling, and many of the same iterative techniques can be exploited. SUPREM-IV uses a conjugate gradient technique with a block preconditioner to achieve speeds up around a factor of two for two-dimensional problems [12].

The second problem concerns three-dimensional grid generation and adaptation. Generation is a problem that has been under study for device simulation [16-18]. Many of these techniques can be adapted for use with process simulation, however, it is important to consider refinement techniques for process simulation since the profile changes during the simulation. The initial grid may not be adequate for the final structure. Lin et al., have developed error estimators for diffusion simulation that may help with this problem [19].

The main challenge for grid generation is the adaptation that must occur due to 
the moving oxide/silicide interface. Oxidation and silicidation are natural companion processes to dopant diffusion simulation, and therefore the grid must be adapted to conform to the growing layer driven by surface reactions. This represents a key problem for $2 \mathrm{D}$ simulation reliability, as a large number of the problems with current versions of SUPREM-IV come from the grid adaptation algorithm. Some encouraging $2 \mathrm{D}$ results have been presented $[20,21]$, but further work will be required for threedimensional simulation.

\subsection{Cultural Barriers}

The simplest answer to the lack of three-dimensional process simulation is that there are few research labs that are actively pursuing this goal, particularly when compared to the amount of activity in device simulation. Lack of work in the field necessarily produces slower progress. At this conference, for example, 15 of the 80 papers selected for oral presentation concern bulk process simulation. I don't believe that this ratio is atypical for most process and device simulation conferences, and is even lower at the IEDM conference. The paper ratio accurately reflects the distribution of world wide effort on process modeling as compared to device, applications, and numerical research. Why is there less research activity in the area of process simulation than device simulation?

The first reason is facilities. Obviously, industrial electronics firms have access to advanced fabrication facilities. However, these facilities are not designed to build process modeling test cases, but instead focus on advanced device structures. The lack of dedicated facilities for experimental work impedes progress. Another barrier is the perceived difficulty in obtaining results. Rather than using resources to characterize and model a particular process, a series of shotgun experiments is run to optimize the structure. In the short term, this approach is probably effective in reducing costs and development time. In the long run, however, little is learned that can be reused for the next generation of technology. Only the largest companies, to date, have been able to afford to invest in process modeling.

On the other hand, most university research labs do not have extensive fabrication facilities and can not build structures at all. This is in sharp contrast to the situation for device modeling, where transistors are available for measurement from many corporate sources. Fabrication facilities are beyond the means of most universities, and it is increasingly difficult to support those universities that do have facilities. A large fraction of the university based process modeling done in the last decade has come from Stanford and Duke, both of which have large, expensive fabrication facilities. This lack of facilities for experimental work translates into a lack of progress.

Finally, the sheer complexity of the problem discourages researchers. In addition to the diffusion simulation, the flow equations must be treated for oxide and silicide growth in three-dimensions, and accurate calculation of doping profiles from implantation must be obtained. The wide variety of knowledge and disciplines makes it difficult to form an effective process simulation group.

\subsection{Opportunity - Software Engineering}

Figure 2 illustrates the organization of most of the core of process simulation tools. For a complete tool, user interface and post processing tools are required, but these will be ignored in this discussion. There are three major components; grid, physics, and linear algebra. The grid and linear algebra are directly related to the challenges discussed in section 2.3, and the physics relates to the challenges in section 2.2. 


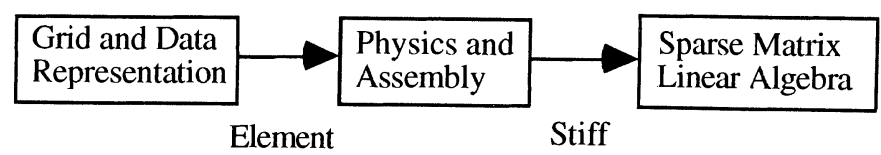

Figure 2: Basic organization of the core of process simulation tools.

These code sections have fairly well defined interfaces that make it possible to organize and write them independently. Object-oriented languages are a natural development framework for this data encapsulation. The code components communicate through elements and stiffness matrices. The physics component needs to be able to assemble the Jacobian for the required PDE's for each element in the mesh. In two-dimensional simulation, the element would be a triangle and for three-dimensional simulation the elements could be either tetrahedra or bricks. The physics code produces a small, dense Jacobian matrix usually referred to as the stiffness matrix for historical reasons. The dense stiffness matrices can then be copied or summed into the matrix component solver one by one to assemble the large sparse matrix.

If a code is structured in this fashion, it is possible for a diverse community to be actively developing the final software package. Mathematicians can work on sparse matrix techniques and insert their code with advanced process models and physics. New grid generation schemes can be implemented at the other end of the scale, without affecting the remaining code components. This has the potential for accelerating development of a three-dimensional process simulator, because each of the major challenge areas can be addressed cooperatively by a wide range of groups in both academic and industrial environments. This in turn tends to lower the structural barriers present to development of a three-dimensional process simulator.

Finally, three-dimensional process simulation will be a computationally expensive undertaking. Intelligent systems need to be developed to manage the simulation of an entire process flow and device structure. The entire structure does not need to be simulated by a three-dimensional code, and many parts can be handled adequately by a one- or two-dimensional code. Such a system is under development that builds 3D device structures from mask information and one-, two-, and three-dimensional process simulation [22]. Such a tool will be required to perform efficient simulation of full device structures.

\section{Conclusions}

There are four major challenges to obtaining accurate three-dimensional process simulators, metrology, models, numerics, and structural. Metrology is the major stumbling block, because it is very difficult to measure two-dimensional profiles, much less three. Model development, particularly phenomenological models that would be more computationally efficient, are slowed due to the lack of sophisticated measurement techniques. These two factors contribute to lack of motivation for development, since without accurate, verifiable models three-dimensional process simulators "garbage in, garbage out."

Numerical problems also provide a major hurdle to development. The first problem is efficient inversion of the large sparse linear system. This problem is similar to the problem faced by device simulation in three-dimensions and can be attacked by 
some of the same methods. The other problem is grid generation and adaptation. Adaptation is required so that diffusion and oxidation/silicidation problems can be solved at the same time. There has been little work on this topic for three-dimensional simulation.

Finally, there is widespread lack of effort in part due to lack of facilities and apparent benefit for development of three-dimensional process simulation. Overcoming the first three hurdles will help make three-dimensional process simulation more attractive. A possible solution to some of these problems is dividing the program into a set of welldefined interfaces between major modules, so that co-development at several locations is possible. This would allow greater leverage to be applied to the problem, and lower the individual cost of development.

\section{Acknowledgements}

I would like to thank the National Science Foundation, SEMATECH, IBM and Silvaco for support of my process modeling research. I would also like to thank Kevin S. Jones and Heemyong Park for critical reading of this paper.

\section{References}

[1] E.W. Scheckler and A.R. Neureuther, Numerical Analysis of Process and Devices Workshop, Seattle, p. 9, 1992.

[2] J.J. Helmsen, E.W. Scheckler, A.R. Neureuther and C.H. Sequin, Numerical Analysis of Process and Devices Workshop, Seattle, p. 3, 1992.

[3] J. Slinkmann, Private Communication.

[4] R.W. Dutton and M.R. Pinto, Proceedings of the IEEE, 74 (12), 1986.

[5] P.B. Griffin and J.D. Plummer,International Electron Devices Meeting, Los Angeles, p. 522, 1986.

[6] R. Subrahmanyan, H.Z. Massoud and R.B. Fair, Appl. Phys. Lett., 52, p. 2145, 1988.

[7] R. Subrahmanyan, H.Z. Massoud and R.B. Fair, J. Electrochem. Soc., 135, p. 1573, 1990.

[8] S. Goodwin-Johannson, R. Subrahmanyan, C.E. Floyd and H.Z. Massoud, IEEE Trans. CAD, 8(4), p. 323, 1989.

[9] N. Khalil and J. Faricelli, Simulation of Semiconductor Devices and Processes, Vienna, 1993.

[10] R.B. Fair and J.E. Rose, International Conference on Computer Aided Design, Santa Clara, 1987.

[11] M.E. Law, C.S. Rafferty and R.W. Dutton, International Electron Devices Meeting, Los Angeles, p. 518, 1986.

[12] M.E. Law and R.W. Dutton, IEEE Trans. on CAD, 7(2), p. 181, 1988.

[13] C. Boit, F. Lau and R. Sittig, Applied Physics A, 50, p. 197, 1990.

[14] G.B. Bronner and J.D. Plummer, J. Appl. Phys., 61(12), p. 5286, 1987.

[15] P.B. Griffin, P.M. Fahey, J.D. Plummer and R.W. Dutton, Appl. Phys. Lett, 47(3), p. 319, 1985.

[16] J. Fuhrmann and K. Gärtner, Simulation of Semiconductor Devices and Processes, Vienna, 1993. 
[17] N. Hitschfeld and W. Fichtner, Simulation of Semiconductor Devices and Processes, Vienna, 1993.

[18] N. Hitschfeld, P. Conti and W. Fichtner, Simulation of Semiconductor Devices and Processes, p. 165, 1991.

[19] C.C. Lin, M.E. Law and R.E. Lowther, IEEE Trans. CAD, 12(10), 1993.

[20] Z.H. Sahul, R.W. Dutton and M. Noell, Simulation of Semiconductor Devices and Processes, Vienna, 1993.

[21] K. Smith and R.E. Bank, NUPAD, Seattle, p. 187, 1992.

[22] C. Hegarty, T. Feudel, N. Hitschfeld, R. Ryter, N. Strecker, M. Westermann and W. Fichtner, Process Physics and Modeling in Semiconductor Technology Symposium, Honolulu, p. 565, 1993. 